Article

\title{
Low-Cost High-Speed In-Plane Stroboscopic Micro-Motion Analyzer
}

\author{
Shashank S. Pandey *, Aishwaryadev Banerjee, Mohit U. Karkhanis and Carlos H. Mastrangelo \\ Department of Electrical and Computer Engineering, University of Utah, Salt Lake City, UT 84112, USA; \\ devaash88@gmail.com (A.B.); mohit.karkhanis@utah.edu (M.U.K.); carlos.mastrangelo@utah.edu (C.H.M.) \\ * Correspondence: shashank.s.pandey@utah.edu; Tel.: +1-801-587-7587
}

Received: 13 November 2017; Accepted: 30 November 2017; Published: 30 November 2017

\begin{abstract}
Instrumentation for high-speed imaging and laser vibrometry is essential for the understanding and analysis of microstructure dynamics, but commercial instruments are largely unaffordable for most microelectromechanical systems (MEMS) laboratories. We present the implementation of a very low cost in-plane micro motion stroboscopic analyzer that can be directly attached to a conventional probe station. The low-cost analyzer has been used to characterize the harmonic motion of $52.1 \mathrm{kHz}$ resonating comb drive microactuators using $~ 50 \mathrm{~ns}$ pulsed light-emitting diode (LED) stroboscope exposure times, producing sharp and high resolution $(\sim 0.5 \mu \mathrm{m})$ device images at resonance, which rivals those of several orders of magnitude more expensive systems. This paper details the development of the high-speed stroboscopic imaging system and presents experimental results of motion analysis of example microstructures and a discussion of its operating limits. The system is shown to produce stable stroboscopic LED illumination to freeze device images up to $11 \mathrm{MHz}$.
\end{abstract}

Keywords: high speed imaging; stroboscope; vibration mode measurement

\section{Introduction}

Resonating microelectromechanical systems (MEMS) devices have applications in many fields, such as inertial navigation, medical imaging, defense, aviation and avionics, and micro-robotics [1]. Measurements of the device dynamics are essential for the understanding and analysis of vibration eigenmodes. Several dynamic motion measurement techniques have been reported and broadly classified into two categories; (a) real time and (b) time-averaged techniques [2]. Real time techniques, such as Laser Doppler Vibrometry (LDV), have been extensively used over the years [3]. LDV performs the dynamic analysis of the surface under examination by extracting the Doppler frequency shift of a reflected laser beam one spot at a time. The non-contact nature and high precision of this technique makes it attractive for the characterization of MEMS devices. However, the real-time nature of the measurement requires very high-speed recording of measurements and expensive electro-optical systems.

In recent times, optical microscopy coupled with stroboscopic (pulsed) time-averaged imaging has attracted a lot of attention for imaging rigid body vibrations as an alternative to LDV [4-12]. Several researchers have reported in-plane, out-of-plane, and full three-dimensional (3D) motion analysis of MEMS structures using stroboscopic imaging coupled with interferometry [4,5]. A major advantage of using pulsed light is that this technique can produce 'freeze' images of high speed device motion with low-frame rate digital complementary metal-oxide-semiconductor (CMOS) cameras; hence, it can measure the motion of microstructures that are vibrating at $\mathrm{MHz}$ frequencies at a lower cost than LDV. Further use of digital image post processing techniques has also enabled researchers to analyze microstructure motion at very high resolution in the order of a few nanometers $[4,8]$. 
Both LDV and stroboscopic MEMS device motion analyzers are commercially available and are capable of analyzing device in-plane motion of up to 20-25 MHz [13,14]. The pulse width for imaging is $100 \mathrm{~ns}$ for the Polytec MSA-400 system (Polytec Inc., Irvine, CA, USA) [13], which in turn limits the maximum velocity of moving microstructures to be imaged as $10 \mathrm{~m} / \mathrm{s}$. The stroboscopic imaging system designed by us has a capability of imaging with $50 \mathrm{~ns}$ pulse, which is capable of measuring as high as $20 \mathrm{~m} / \mathrm{s}$ velocity of MEMS structure. The Lyncée Tec system (Lyncée Tec., Lausanne, Switzerland) [14] uses a pulsed laser for stroboscopic imaging and has a 7.5 ns pulse imaging pulse width, which is currently the state of the art system available for in-plane motion imaging, but it also costs upwards of $\$ 200,000$. These sophisticated and costly instruments however are largely out of reach for most MEMS researchers. In this paper, we have developed a low cost in-plane motion analyzer which can be attached to a conventional probe station. The system is implemented with a combination of off-the shelf microcontrollers and CMOS logic circuits, a low-light monochrome CMOS camera and a high-power lighting LED pulsed light source. The low-cost system is capable of exposure times of $\sim 50 \mathrm{~ns}$, thus enabling real time synchronous resonance motion measurement of high speed microstructure in the $\mathrm{MHz}$ region. With this system, sharp images of a resonating comb drive at a velocity $\sim 20 \mathrm{~m} / \mathrm{s}$ were captured by a CMOS camera with a resolution of $\sim 0.5 \mu \mathrm{m}$ without the use of any cumbersome post image processing algorithms. Our system is approximately $\sim 98 \%$ less expensive than commercially available solutions that are provided by Polytec and Lyncée Tec [13,14].

\section{Low-Cost Stroboscopy Imaging System}

Figure 1 shows the schematic of the low-cost stroboscopic imaging system. The computer controlled system consists of four major blocks: (i) the synchronous timer circuit, (ii) the LED switching circuit, (iii) the high voltage comb driver circuit, and (iv) a conventional probe station imaging microscope with a low-light CMOS digital camera. The imaging system produces a periodic sharp pulse of light from a high-power LED that is synchronized with the device drive, thus producing stationary images of the oscillating device, provided that the device motion is periodic and the period being equal to the ratio of the excitation signal period divided by an integer factor. Stroboscopic systems such as this require precise timing and synchronization of all the signals that are produced by a timer generator circuit.

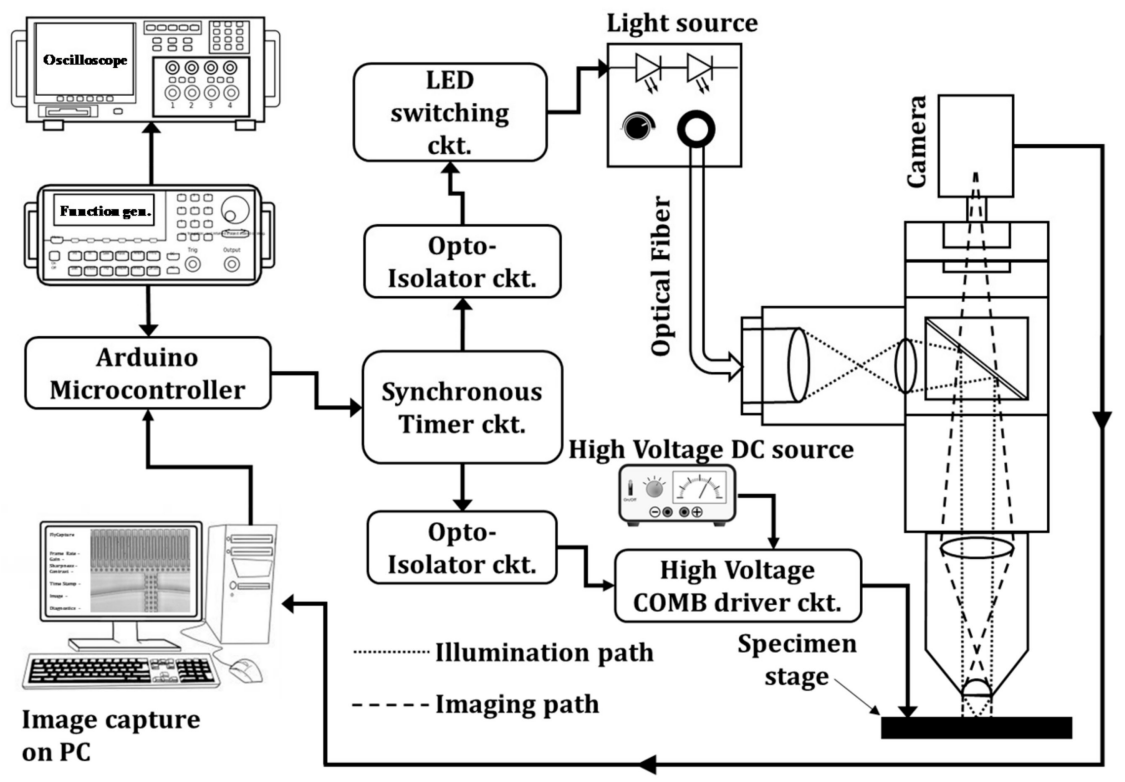

Figure 1. Block diagram of stroboscopic imaging system. 


\subsection{Timer Generator Circuit}

This circuit produces all of the synchronized signals that are necessary for driving the device and LED strobe. In time-averaged stroboscopic imaging, the MEMS device is driven by a periodic signal at the desired excitation frequency. The device position at a given phase is captured by the CMOS camera by flashing a very narrow but bright pulse of light with a known delay from the leading edge of the drive signal. Figure 2 illustrates a schematic plot of phase relations between the drive and LED pulses, and the corresponding freeze image for a vibrating comb finger example. Since the motion of the MEMS device is periodic, the recorded image is stationary, and the image brightness that is recorded by the camera is increased simply by integration over many of these pulses. It is also important to note that since the camera integrates the image over the duration of each pulse; the LED pulse should be as sharp as possible to prevent any image smearing.
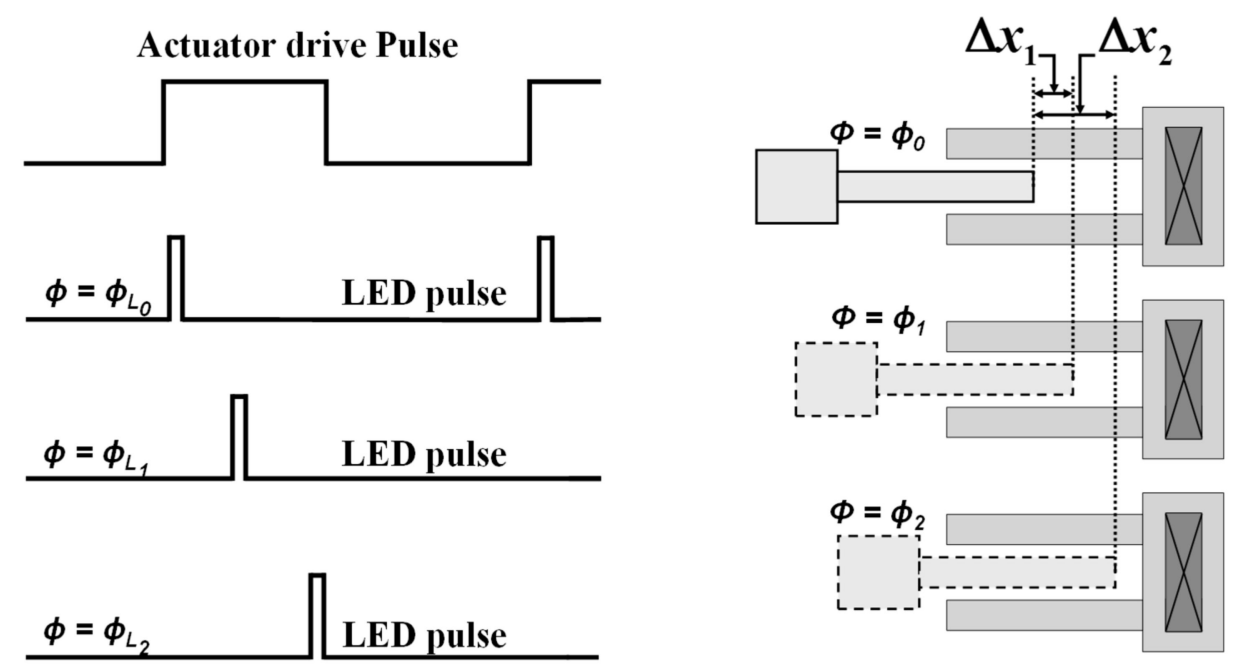

Figure 2. Schematic representation of stroboscopic imaging by phase delayed imaging light-emitting diode (LED) pulse.

In order to image the device motion at different times, it is necessary to vary the phase delay $\phi$ of the strobe pulse. The delayed and narrow LED strobe pulse can be generated using different circuit schemes. A common method uses tunable analog delay lines or analog monostable multivibrators. The main disadvantage of analog delay schemes is that the LED pulse phase would change when the drive frequency is swept. This phase however does not change if the LED pulse is generated digitally. For example, one could generate a strobe pulse with a modulo- $M$ digital counter every time that the count reaches a particular value $N_{L}<M$. High-precision periodic digital strobe signals can be generated with high-speed finite-state machines using programmable gate arrays or high-speed transistor-transistor logic (TTL) or CMOS logic chips. In this paper, we utilize a TTL implementation due to its simplicity and low cost.

Figure 3 shows the schematic of the digital timer generator block diagram. It utilizes two types of digital modules. The first module produces a programmable modulus period signal $(P P)$ of frequency $f_{c} / M$. The second module is a digital one-shot circuit that produces a single delayed pulse at a specified count $N_{i}$ starting from the rising edge of the START input. For example, the phase delay for such a digital pulse is thus $\phi_{i}=2 \pi N_{i} / M$ radians. 


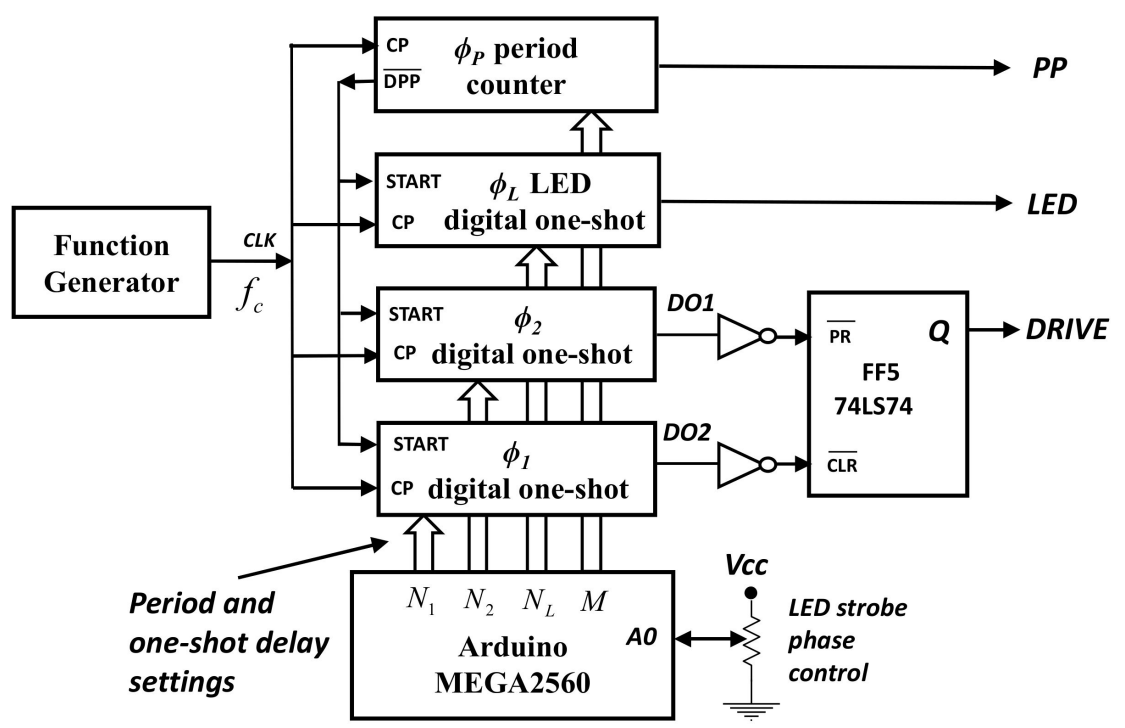

Figure 3. Schematic diagram of the timer circuit showing the generation of the output pulses using the digital delay modules.

In order to produce the $P P$ period, LED pulse and DRIVE signals we utilize one period module and three digital one-shot modules. Two of the digital one-shots are used to signal the beginning and end of the DRIVE signal within the period PP. The value of each $N_{i}$ is loaded into the period and one-shot modules using an Arduino Mega 2560 microcontroller (Digi-Key Electronics, Thief River Falls, MN, USA). These values are set by the Arduino programming code and the digitized voltage at the Arduino input $\mathrm{A}_{0}$ that is provided by a phase control potentiometer. Figure 4 shows the timing diagrams of the resulting digital timing signals.

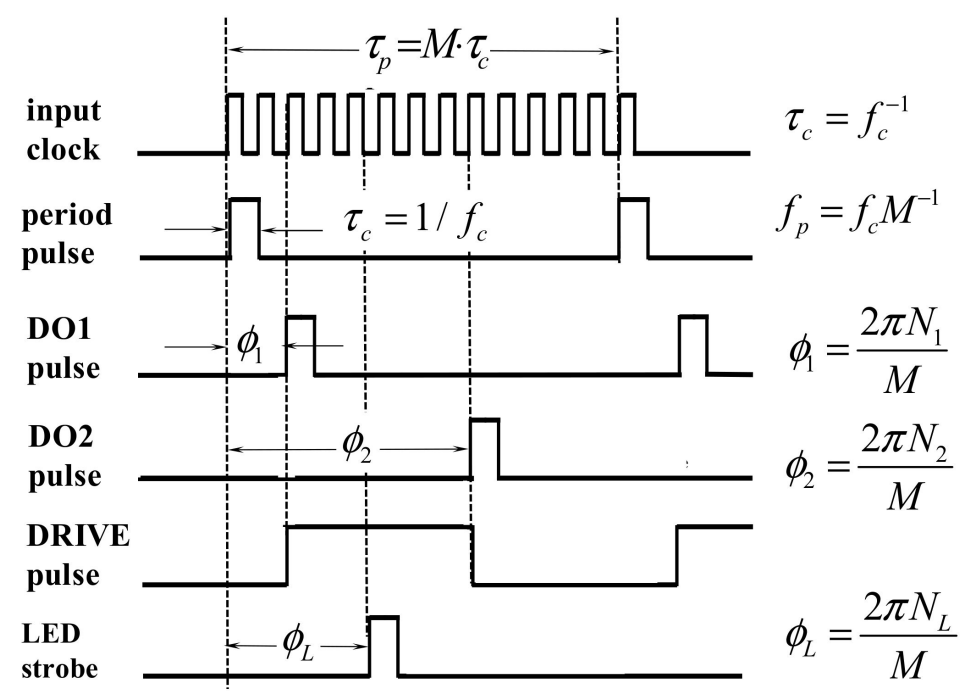

Figure 4. Schematic logic diagram indicating generation of both strobe and drive signals from four narrow digital delay pulses at different digital phase $\phi_{0}-\phi_{3}$.

Figure 5a shows the implementation of the period counter module using a 74HCT4059 CMOS programmable divide-by-n counter and $\frac{1}{2}$ of a 74LS74 D flip-flop. The counter chip first loads a two-digit binary-coded decimal (BCD) digital number between 0 and 99 at the counter J1-J8 JAM inputs representing the number of clock pulses and the period length. Then it subsequently counts down to zero and produces an output pulse $(P P)$. The counter next loads the BCD number again, 
and repeats the cycle. A digitally delayed and inverted period pulse $(\overline{D P P})$ is also generated through the D flip-flop.

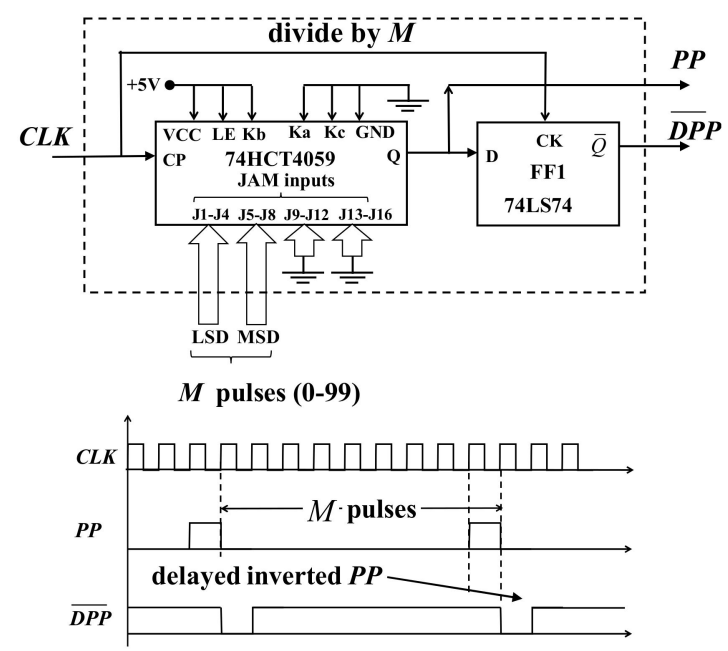

(a)
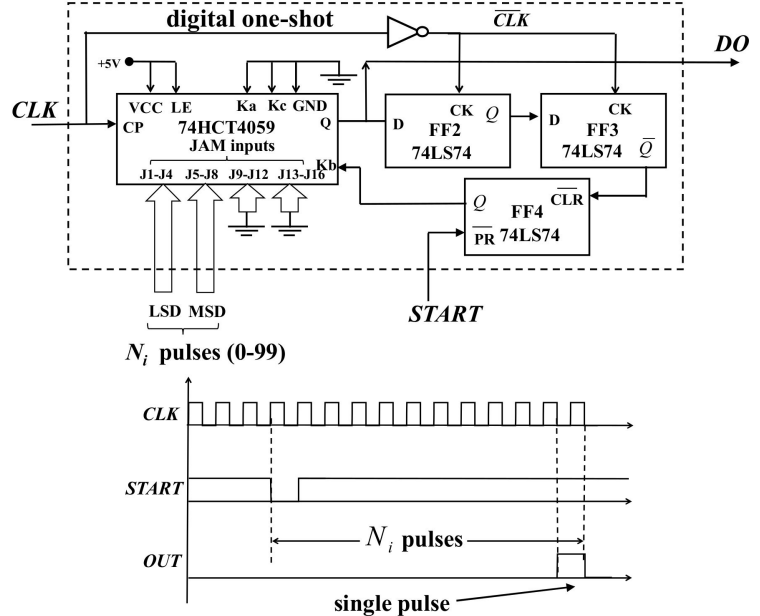

(b)

Figure 5. (a) Schematic and timing diagram of the period module used as period pulse generator. The period module produces a repeating pulse every $N_{i}$ input pulses. (b) Schematic and timing diagram of digital one-shot module. The digital one-shot produces a single delayed pulse after the START signal goes high.

Figure $5 \mathrm{~b}$ shows the digital one-shot module. This module has two inputs, a clock pulse from the function generator and a START pulse, which is same as the $\overline{D P P}$ output pulse from the period counter. The one-shot module produces a single pulse delayed at $\left(N_{D}\right)<M$ that is triggered by a high logic level at the START input. The one-shot consists of a 74HCT4059 counter [15] (NXP Semiconductors, Eindhoven, Netherlands), 2D flip-flops and 1 RS flip-flop. The down counter is configured such that the counter initial value $N_{D}$ is first loaded while the $k_{b}$ control pin is at logic low. The counter remains in the loading state until the state of FF4 is changed. When the START signal goes to logic low, it presets FF4 and the $k_{b}$ control pin goes to logic high and the counter starts counting down. When the count reaches zero its $Q$ output produces a $D D$ pulse, which is subsequently delayed by FF2 and FF3 and used to clear flip-flop FF4 setting the counter loading mode until a new START signal again goes to logic low, restarting the single-shot cycle.

\subsection{High Voltage MEMS and LED Driver Circuits}

These circuits convert the low voltage digital signal from the timer circuit to a high voltage and high-power signals that are needed to drive the MEMS devices and the LED. Figure 6a shows a schematic of the MEMS and LED driver circuits. High voltage signals needed to drive electrostatic MEMS actuators are generated using a L6384E (STMicroelectronics, Geneva, Switzerland) half bridge gate driver chip connected to two high speed, high voltage power metal-oxide-semiconductor field-effect transistor (MOSFETs) (STF2N80K5, STMicroelectronics, Geneva, Switzerland) M1 and M2. This configuration enables the output to switch from 0 to a specified high voltage. The input of the half-bridge is connected to the TTL timer circuit through an optoisolator (6N137, Lite-On, Taipei, Taiwan) which prevents any high voltage back feed onto the timer circuit. The half bridge driver circuit works up to a maximum frequency of about $0.5 \mathrm{MHz}$. 

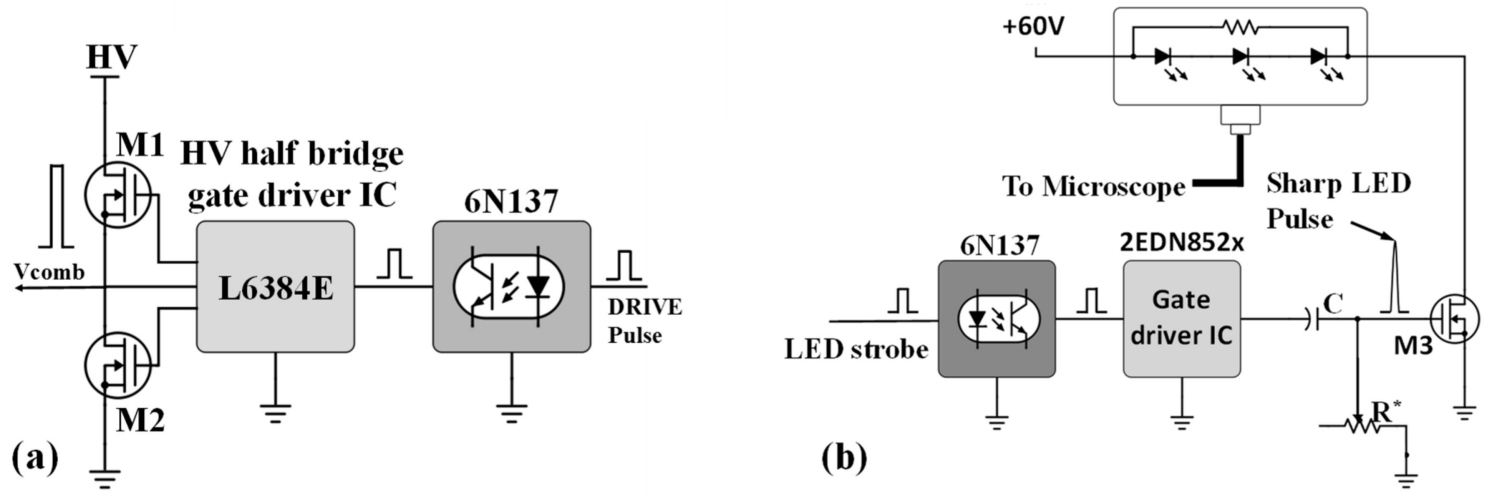

Figure 6. (a) Schematic of the half bridge gate driver configuration of high voltage MEMS driver ckt. and (b) schematic of the high-speed LED switching ckt. using an ultrafast gate driver integrated circuit (IC) with an $\mathrm{R}^{*} \mathrm{C}$ differentiator ckt. for producing sharp LED pulses.

Figure $6 \mathrm{~b}$ shows the LED pulse generation circuit. The LED signal from the timing generator drives an optoisolator (6N137, Lite-On, Taipei, Taiwan) and a high-current power MOSFET driver chip (2EDN852x, Infineon Technologies, El Segundo, CA, USA). This driver chip can produce a peak transient gate currents of 1.5 A that is necessary for high speed MOSFET switching ( 20 ns). A high-lumen lighting LED (CXM-32 series high lumen LED from Luminus (Sunnyvale, CA, USA) connected to the drain of M3 is connected to an illumination fiber is used to provide pulsed light. The duration of LED light pulse width is controlled by an $\mathrm{R}^{*} \mathrm{C}$ differentiator circuit connected to the gate of M3 which makes the pulse sharper to $\sim 50-80 \mathrm{~ns}$.

\subsection{Imaging Optics}

The LED generated a cool white light that was centered at a wavelength $\lambda=450 \mathrm{~nm}$ with an output flux of 18,820 lumens. The strobed LED light is introduced into an epi-illuminated microscope (Mitutoyo WF series) with a Mitutoyo Plan Apo infinity corrected long working distance (WD) objective. The magnification on the lens objective was 20x with a numerical aperture (NA) of 0.42 . Optical resolution for this set-up can then be estimated by using Abbe's limit $(R=0.5 \lambda / N A)$, which gives a resolution of $\sim 0.5 \mu \mathrm{m}$. Any motion below this value cannot be observed optically and requires image post processing. The freeze frame image is recorded by a c-mount 14-bit 2.8 MP monochromatic Grasshopper 3 series camera from FLIR Integrated Imaging Solutions Inc. (Wilsonville, OR, USA) with a pixel size of $3.69 \mu \mathrm{m} \times 3.69 \mu \mathrm{m}$. The high quantum efficiency and low temporal dark noise level on this camera sensor generates an image with high signal to noise ratio. In order to obtain a faithful image from the camera, at least three pixels need to be excited i.e., an image size of $\sim 11 \mu \mathrm{m}$ on the sensor which back annotated to the device under test (DUT) for a 20x magnification objective means that a movement of $\sim 0.55 \mu \mathrm{m}$ can be sensed by the camera. Since this value is about the same as the optical resolution of the microscope objective, the camera itself does not put any constraint on the image resolution. As high as 26 frames per second (FPS) can be measured with this camera. For stroboscopic freeze-frame imaging system where capturing a static image does not require a high FPS, instead, using a low frame rate (3 FPS) increases the image brightness. The real-time image from the camera is displayed on a PC using the FlyCapture software interface from FLIR Integrated Imaging Solutions Inc. (Wilsonville, OR, USA). The total cost of the timer generator, MEMS and LED drive circuits and imaging system excluding the microscope and external signal generators was $\angle$ \$2000 USD.

\section{Test Device Fabrication}

We have used electrostatic comb drive devices for testing the stroboscopic imaging system. Figure 7 shows a simplified fabrication scheme for the comb drive microactuators. Devices were 
fabricated on a silicon on insulator (SOI) wafer with $30 \mu \mathrm{m}$ device layer and $1 \mu \mathrm{m}$ buried oxide using a single mask process. The comb features were etched on the wafer through High Frequency deep reactive ion etching (DRIE) for first $25 \mu \mathrm{m}$ ending with a low frequency etch for the last $5 \mu \mathrm{m}$ to avoid any footing at the buried oxide layer, using positive photoresist as etch mask. Following the DRIE etching, the comb drives were released by etching the buried oxide (BO) in a 5:1 BHF bath. Etched release holes on the wide features, such as main shuttle and trusses, enabled a faster release to prevent anchors from coming off. The HF dip was then diluted with a large quantity of deionized (DI) water. The DI water is then replaced with methanol by dilution. All of this while the die is kept inside the solution in order to prevent stiction of the released structures with the substrate underneath. Device dies were then supercritically dried. Figure $8 \mathrm{a}, \mathrm{b}$ shows the schematic of the comb drive actuator with all of the important dimensions and Figure $8 \mathrm{c}$ shows the SEM images of the released devices.
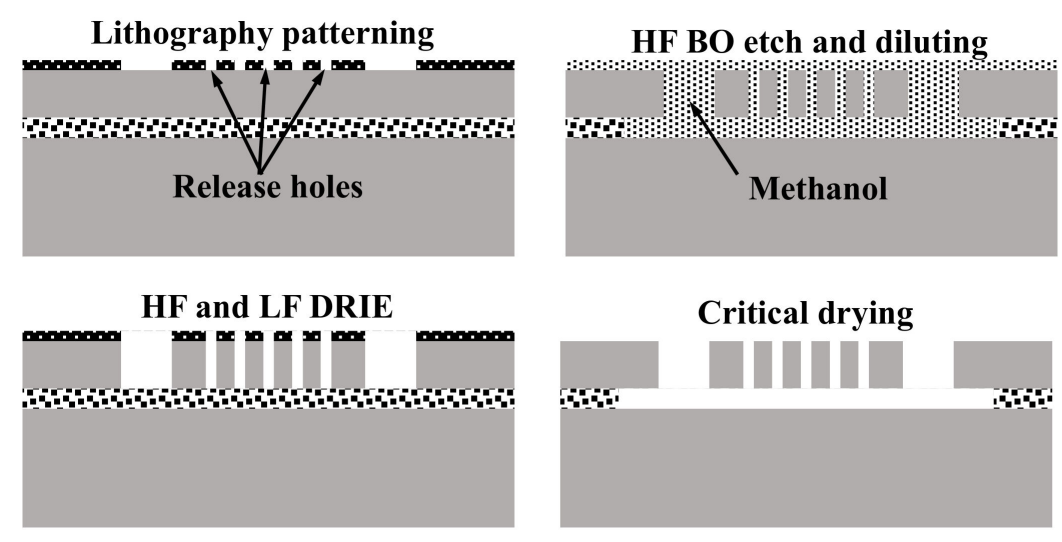

Figure 7. Simplified fabrication process of test comb drive microstructures.

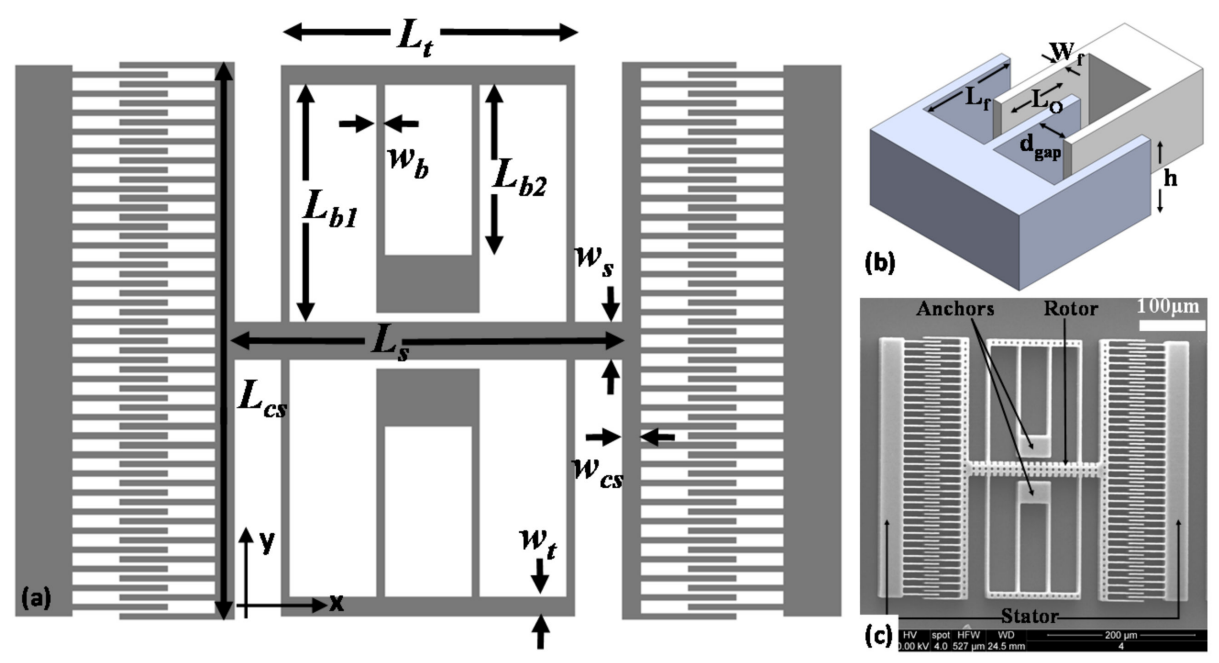

Figure 8. (a) Two-dimensional (2D) schematic of the COMB drive actuator with critical dimensions labeled. (b) 3D schematic of the COMB drive actuator with the rest of the critical dimensions labeled and (c) SEM photographs of the released COMB drive microactuator used for testing.

\section{Comb Drive Motion}

The motion of the comb drive is governed by the second order differential equation for forced oscillations of a spring mass damper system, as given by the Equation (1) and shown in Figure 9.

$$
m_{e f f} \ddot{x}+b \dot{x}+k_{e f f} x=F_{e x t}(t, \omega)
$$


where $m_{\text {eff }}$ is the effective mass of the system under motion, $b$ is the damping coefficient, $k$ is the net stiffness constant for the folded beam system, $F_{\text {ext }}$ is the electrostatic excitation force which is a function of time $(t)$ and frequency $(\omega)$, and $x$ is the displacement in the $x$ direction. The excitation force is the result of fluctuating electrostatic voltage from the high voltage comb drive pulse train and it can be derived from the rate of change of energy stored in the capacitor formed between a rotor finger and two stator fingers and is expressed as $F_{\text {ext }}=\left(n_{f} \varepsilon_{o} h\right) V_{\text {pulse }}^{2} / d_{g a p}$, where $n_{f}$ is the total number of fingers, $\varepsilon_{o}$ is the vacuum permittivity, $h$ is the height of the stator and rotor fingers, $d_{g a p}$ is the gap between the stator and rotor fingers, and $V_{\text {pulse }}$ is the voltage pulse train output from the high voltage driver circuit. To preserve the linearity of the equation of motion, we neglect the sideways movement. The periodic voltage pulse train can be expressed as a Fourier series, as given by Equation (2).

$$
V(t)=V_{o}\left[\frac{\tau}{T}+\sum_{n=1}^{\infty} \frac{2}{n \pi} \sin \left(\frac{n \pi \tau}{T}\right) \cos \left(\frac{2 n \pi}{T}\left(t-\frac{\tau}{2}\right)\right)\right]
$$

where $V_{o}$ is the amplitude of voltage pulse, $\tau$ is the pulse width, and $T$ is the time period which is equal to $2 \pi / \omega$. The effective mass of the moving rotor can be estimated by applying the principle of conservation of energy on the resonating structure and assuming that all of beams deflect with mode shapes as if under static loads [16]. The energy conservation and beam theory together give the effective mass of the resonator as:

$$
m_{e f f}=m_{s}+m_{f}+\frac{12}{35} m_{b}+\frac{1}{4} m_{t}
$$

where $m_{s}$ is the mass of the shuttle, $m_{f}$ that of moving fingers, $m_{b}$ represents mass of eight parallel beams, and $m_{t}$ is the mass of two trusses. Assuming that the trusses act as rigid support for the beams under deflection, the total stiffness for the folded beams under no residual stress can be calculated by using the series and parallel beam theory. Since each beam length is not identical the effective stiffness along $x$ axis becomes:

$$
k_{e f f}=4 \cdot\left(\frac{k_{x, b 1} \cdot k_{x, b 2}}{k_{x, b 1}+k_{x, b 2}}\right)
$$

where $k_{x, b 1}$ and $k_{x, b 2}$ are the beam stiffness for beam elements $b_{1}$ and $b_{2}$, as shown in the figure. From Euler beam theory the stiffness of each beam segment is $k=12 E_{S i} I_{z, b} / L^{3}$, where $E_{S i}$ is the Young's modulus of silicon, $I_{z, b}$ is the area moment of inertia along the $z$ axis, and $L$ is length of the beam segment. The area moment of inertia for beam cross section is given by $I_{z, b}=h w^{3} / 12$, where $h$ is the height, $w$ is the width of the beam. The material properties that were used to calculate the variables in the equation of motion are given in Table 1 below. Using these values, the effective mass of the rotor and the total stiffness for the folded beam arrangement is calculated to be $1.23 \mu \mathrm{g}$ and $210 \mathrm{~N} / \mathrm{m}$, respectively which give the analytical resonant frequency $f_{n}=\sqrt{k_{e f f} / m_{e f f}} / 2 \pi$ as $65.7 \mathrm{kHz}$.

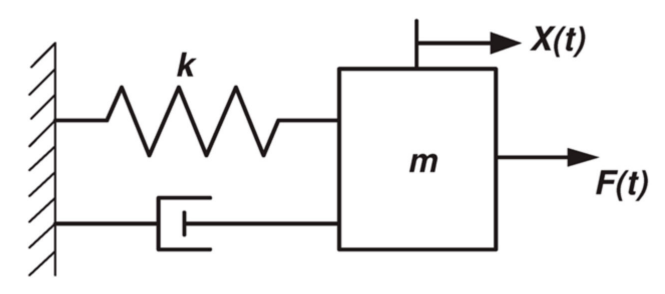

Figure 9. Spring mass damper lumped model equivalent of the comb drive actuators. 
Table 1. Dimensional and material properties for test comb drive devices.

\begin{tabular}{cccc}
\hline Name & Parameter & Value & Unit \\
\hline Shuttle length & $L_{s}$ & 294 & $\mu \mathrm{m}$ \\
Truss length & $L_{t}$ & 153 & $\mu \mathrm{m}$ \\
Width shuttle & $w_{s}$ & 20 & $\mu \mathrm{m}$ \\
Width truss & $w_{t}$ & 10 & $\mu \mathrm{m}$ \\
1st beam length & $L_{b 1}$ & 125 & $\mu \mathrm{m}$ \\
2nd beam length & $L_{b 2}$ & 90 & $\mu \mathrm{m}$ \\
Beam width & $w_{b}$ & 2.5 & $\mu \mathrm{m}$ \\
Support length & $L_{c s}$ & 293 & $\mu \mathrm{m}$ \\
Support width & $w_{\mathcal{~}}$ & 10 & $\mu \mathrm{m}$ \\
Finger length & $L_{f}$ & 50 & $\mu \mathrm{m}$ \\
Finger width & $w_{f}$ & 3 & $\mu \mathrm{m}$ \\
Finger gap & $d_{g a p}$ & 2 & $\mu \mathrm{m}$ \\
Finger overlap length & $L_{o}$ & 22 & $\mu \mathrm{m}$ \\
Number of rotor fingers & $N_{f r}$ & 60 & - \\
Number of stator fingers & $N_{f s}$ & 58 & - \\
Finger height & $h$ & 30 & $\mu \mathrm{m}$ \\
Young's modulus for silicon & $E_{S i}$ & 150 & $\mathrm{GPa} \cdot \mathrm{m}$ \\
Density of silicon & $\rho_{S i}$ & 2330 & $\mu \mathrm{m}$ \\
Buffer oxide thickness & $d_{S i O_{2}}$ & 1 & $\mathrm{~m}^{2} / \mathrm{s}$ \\
Kinematic viscosity of air & $v_{a i r}$ & $1.57 \times 10^{-5}$ &
\end{tabular}

\section{Experimental Imaging Results}

Test comb drive microactuators were driven by a high voltage pulse train $V_{\text {СОMB }}$ that was produced by the circuit of Figure $6 a$, and the device mechanical oscillation motion was captured by the microscope camera when illuminated by a stream of LED pulses that were strobed at a specific phase. The maximum comb drive voltage was kept below the side snap-in instability limit. The comb side-snap pull-in voltage [17] was calculated to be $300 \mathrm{~V}$, but when corrected for dimensional errors in fabrication, it is close to $220 \mathrm{~V}$.

\subsection{Frequency Analysis}

The comb actuators were driven with a voltage amplitude of $150 \mathrm{~V}$ over a wide frequency range. The device's mechanical motion was strobe imaged at the excitation frequency at the phase that provides maximum displacement. The images were recorded at three frames per second (FPS). Using a low frame rate for recording gives longer light integration time, thus increasing image brightness and sharpness. Figure 10 shows the image of comb drive actuator frozen in motion at the maximum stroke for a few frequencies. The maximum stroke length data for 31 frequencies from $15 \mathrm{kHz}$ to $80 \mathrm{kHz}$ were plotted against the frequency. The experimental value of the quality factor can be calculated from this figure using the $-3 \mathrm{~dB}$ frequency points in the graph. From this curve, we observed that the natural frequency of the comb drive actuator is $52.1 \mathrm{kHz}$, which is $20 \%$ off from the calculated resonant frequency. This error can be attributed to dimensional errors in the fabricated device and a trapezoidal profile of the DRIE etched features. The effect of these geometric errors has a pronounced impact on the beam stiffness and negligible effect on the effective mass of the system. We calculated the adjusted beam stiffness based on the observed resonant frequency as $k_{a d j}=132 \mathrm{~N} / \mathrm{m}$. The $Q$ factor from the experimental result was 113, which when compared to the calculated analytical value $(\sim 155)$ as given in [18,19], is off by $\sim 27 \%$. Adjusting the $Q$ factor value with the experimentally found $k_{a d j}$ value gives a $Q$ factor of 118 , which is in good agreement with the observed value. The images at peak resonance show that the maximum displacement in $+x$ direction was $\sim 30 \mu \mathrm{m}$, so the total stroke $d_{S}$ of the resonating comb drive was $\sim 60 \mu \mathrm{m}$. 

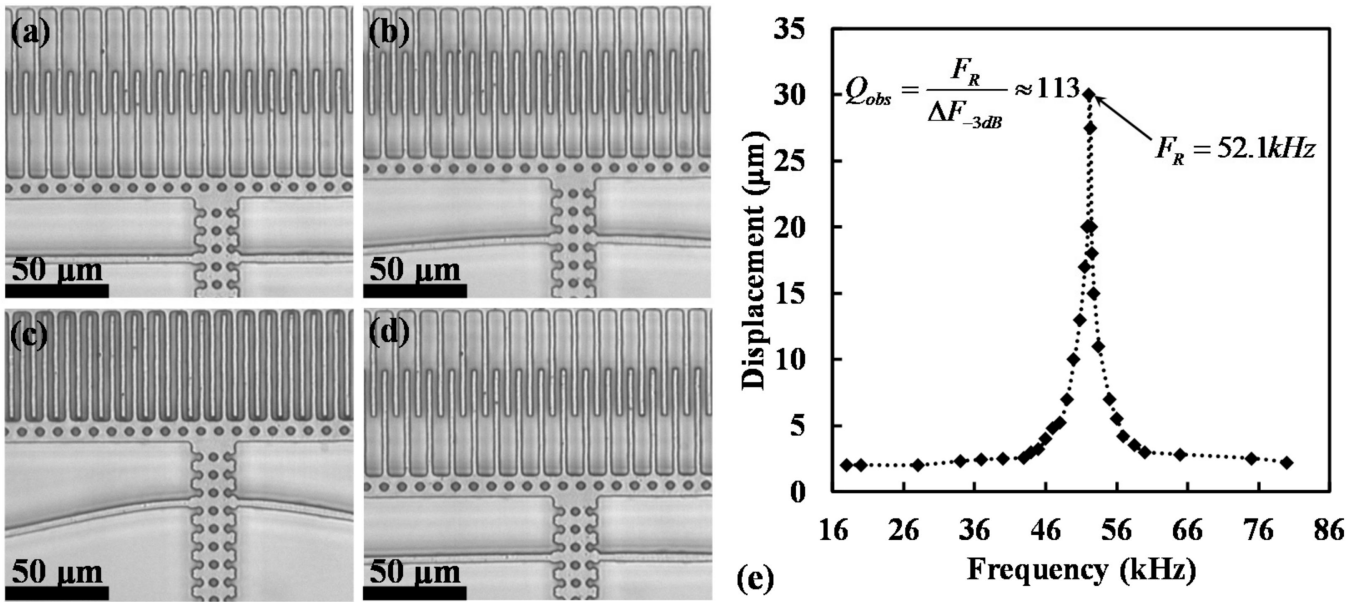

Figure 10. Frequency response through stroboscopic freeze frame images of comb drive actuator at maximum displacements for different frequencies (a) No actuation, (b) $50 \mathrm{kHz}$, (c) $52.1 \mathrm{kHz}$, (d) $80 \mathrm{kHz}$ and (e) Frequency response graph for all of the frequencies tested giving observed $Q$ factor $\approx 113$ and resonance frequency of $52.1 \mathrm{kHz}$.

\subsection{Phase Analysis}

The speed of motion $v=2 \pi F_{R} \cdot d_{s}$ for this comb drive that was calculated from the images was $\sim 20 \mathrm{~m} / \mathrm{s}$. Imaging at such high speed of motion requires a very sharp LED pulse of the order of $\sim 50 \mathrm{~ns}$. This was achieved by adjusting the $R^{*} C$ value in the LED driver circuit. With these sharp LED pulses, the resonance motion of the comb drive was imaged at different phases. Figure 11 shows the images that were captured by our stroboscopic system at different phases. The reference position of the shuttle without any actuation voltage is chosen as $x=0$, and displacement for each phase of motion is approximated from the images w.r.t. the $x=0$ position. This data was plotted, which showed that the motion follows a cosine curve, as was expected from analytical analysis.
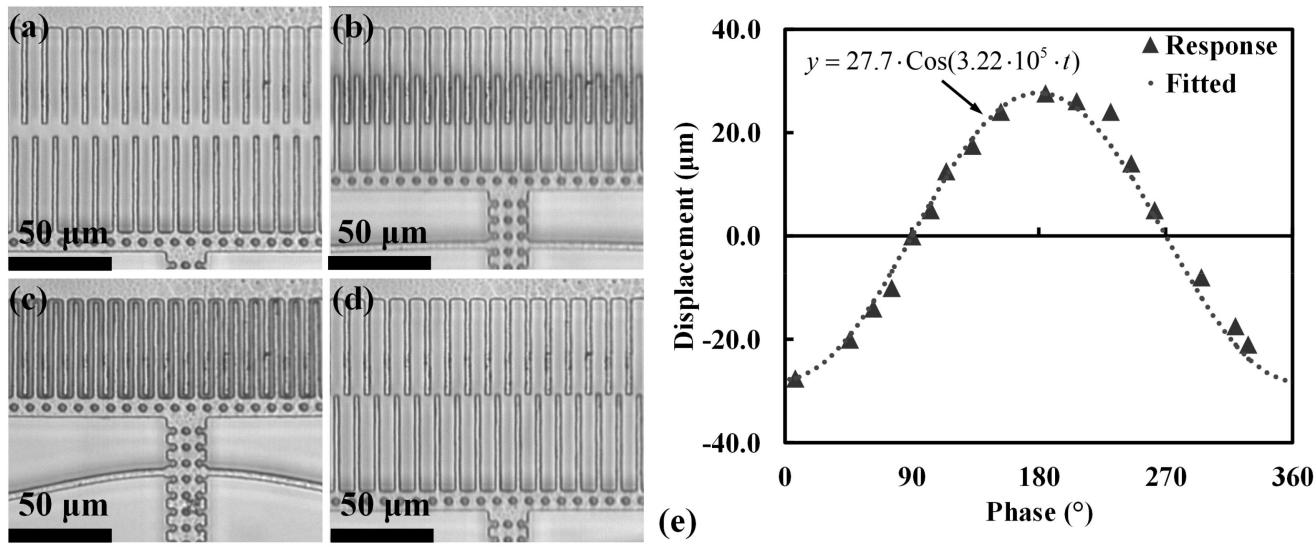

Figure 11. Phase resolved resonant motion of a test comb drive actuator imaged through stroboscopic imaging system at resonance at (a) $7^{\circ}$, (b) $90^{\circ}$, (c) $184^{\circ}$, (d) $330^{\circ}$, and (e) graph of displacement vs. phase w.r.t. the drive pulse shows a cosine relation between displacement and time, which is consistent with the device theory in discussed in Section 4.

\subsection{Stroke vs. Applied Voltage}

The effect of applied voltage to the maximum stroke was also observed, as shown in Figure 12. Note that the stroke length in the plot is twice the maximum displacement that was observed in the 
$+x$ direction. The drive voltage was varied from 40 to $150 \mathrm{~V}$. A square dependence of stroke length on the applied voltage is observed, which is consistent with the comb drive motion theory of Section 4.
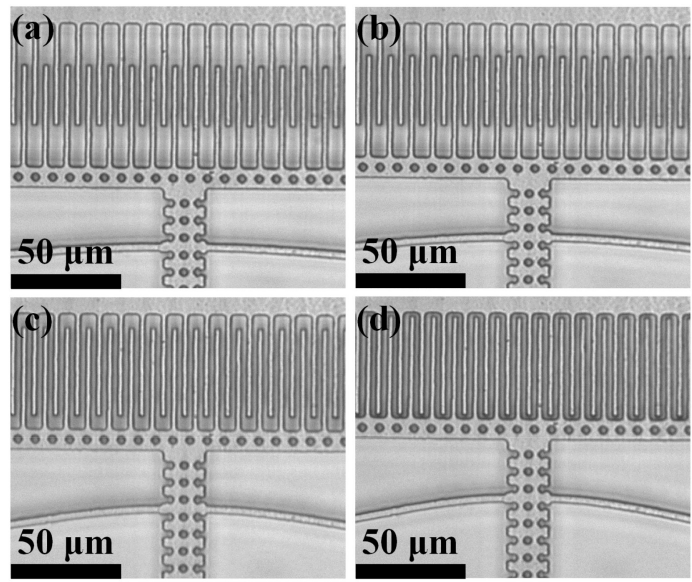

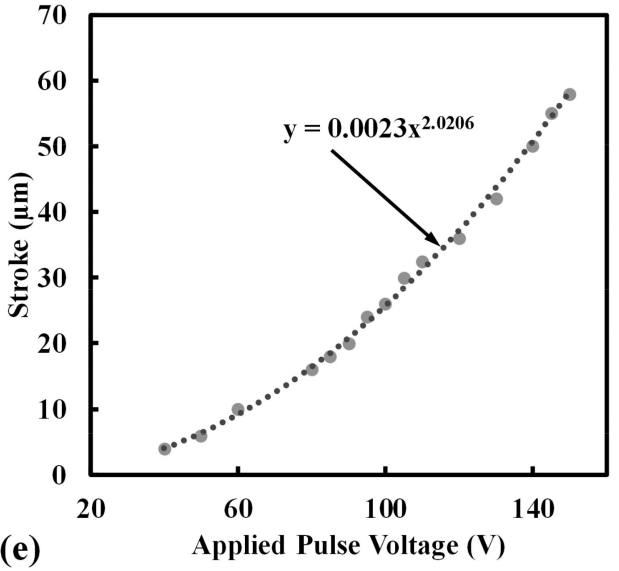

(e)

Applied Pulse Voltage (V)

Figure 12. Effect of applied voltage to maximum displacement observed through stroboscopic imaging set-up at (a) $90 \mathrm{~V}$, (b) $110 \mathrm{~V}$, (c) $130 \mathrm{~V}$, (d) $150 \mathrm{~V}$, and (e) stroke is plotted against the applied voltage, and it was observed to have a square dependence on applied voltage which agrees with the device theory.

\subsection{Stroboscopic System Limitations}

The TTL timer generator implementation that is discussed here has a maximum input clock frequency of about $84 \mathrm{MHz}$ [19], which at modulo factor $M$ of 100 results in a maximum imaging frequency of $840 \mathrm{kHz}$, but other faster schemes are also possible using gate arrays or when operated a lower $M$. If a faster timer generator circuit is utilized, the fundamental limitation is determined by the maximum LED strobe frequency and pulse width. The maximum strobe frequency is limited by the maximum frequency at which the gate driver IC for the LED driver circuit, as shown in Figure $6 \mathrm{~b}$, can work stably. The pulsing of LED was captured on an avalanche photodetector (Thorlabs APD120A2/M-Si Avalanche Photodetector (Thorlabs, Newton, NJ, USA) that was connected to the oscilloscope. The LED pulse and the output from the photodetector was plotted together. The imaging system was observed to work flawlessly up to a strobe frequency of $11 \mathrm{MHz}$, as shown in the Figure 13a. Another constraint on the imaging system is determined by the width of the LED pulse that defines the phase interval of image exposure. A sharp pulse width ensures minimum blurring while capturing a dynamic MEMS actuator which results from a finite amount of movement of the micro-structure during the time for which the LED is on. The pulse width was controlled using an $R^{*} C$ differentiator circuit with a variable resistor such that a pulse width of 50-80 ns was maintained for all of the frequencies. It was observed that below $50 \mathrm{~ns}$ the LED failed turn on or provided very little light, making the frame rate of the camera very low.

The impact of the use of the $R^{*} \mathrm{C}$ differentiator is shown in Figure 13b at a frequency of $52.1 \mathrm{kHz}$. Since we observed the speed at resonance to be $\sim 20 \mathrm{~m} / \mathrm{s}$, an undifferentiated $190 \mathrm{~ns}$ LED on-time generated from the timing generator would results in an image blur of $\sim 3.8 \mu \mathrm{m}$. However, when the pulse width is decreased to $50 \mathrm{~ns}$ with the differentiator, the motion blur of the comb-drive is reduced to $\sim 1 \mu \mathrm{m}$, which is close to the optical resolution of the imaging system $(0.6 \mu \mathrm{m})$. The difference in image clarity on the recorded images at the same phase of motion with and without the differentiator is shown in Figure 13c,d. Note that the differentiated LED signals produces a significant improvement in the image sharpness. 

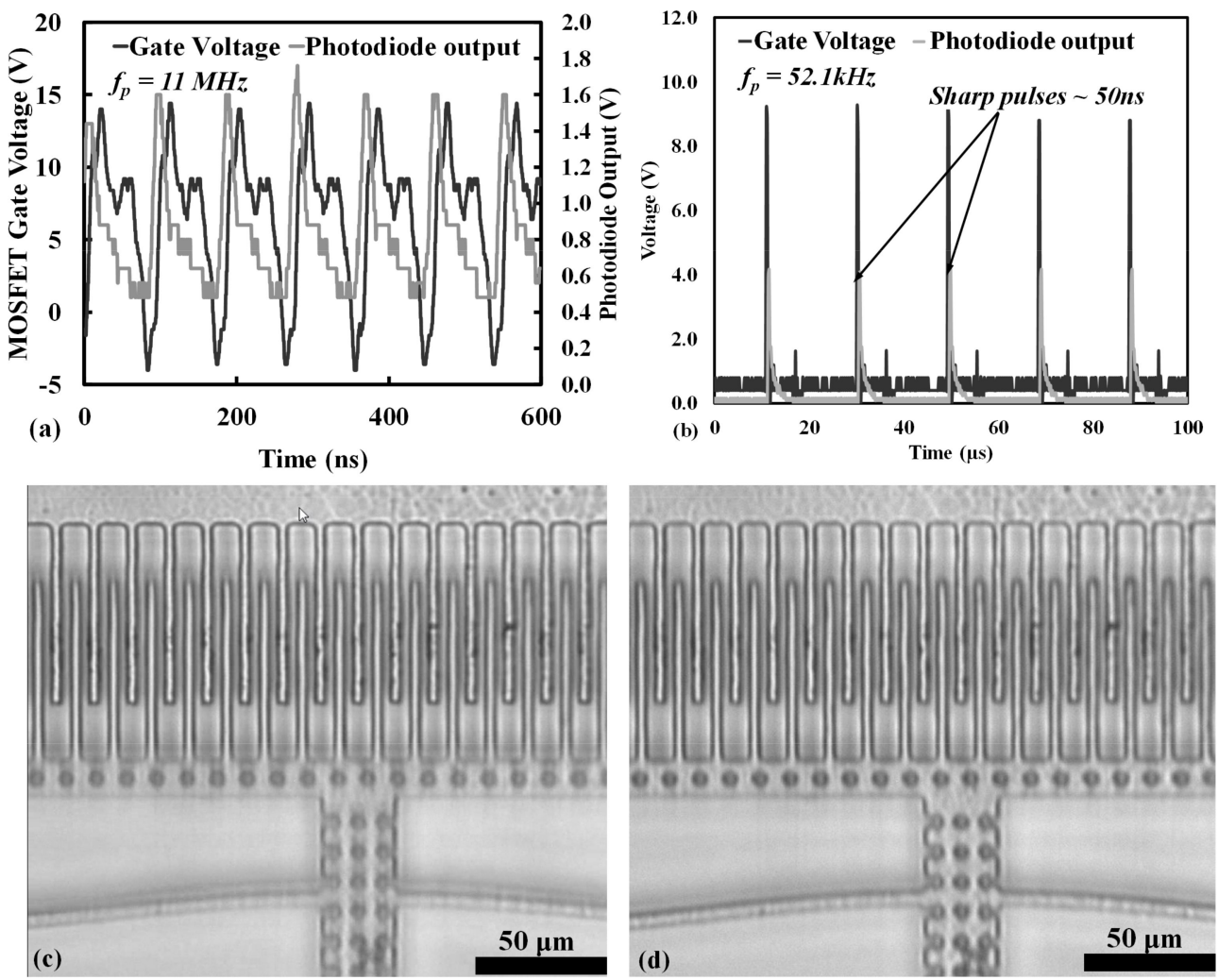

Figure 13. Performance limits of the imaging system. (a) The imaging system is shown to work well at srtobing frequencies up to $11 \mathrm{MHz}$ frequency as shown by the pulsed LED output detected by a high-speed photodetector placed at the camera c-mount; (b) The sharpening of the LED pulses is shown at the resonant frequency of $52.1 \mathrm{kHz}$ with $50 \mathrm{~ns}$ sharp pulses from an $R^{*} \mathrm{C}$ differentiator; Figure (c) shows the motion blurring in the captured image at $52.1 \mathrm{kHz}$ with rectangular $190 \mathrm{~ns}$ strobe pulse widths as achieved from a divide by 100 counter; (d) shows a reduction in motion blurring when the LED signal is sharpened with a differentiation circuit producing $50 \mathrm{~ns}$ wide strobe pulses.

\section{Conclusions}

We have demonstrated the construction, testing, and performance limits of a low cost (USD < \$2000) in-plane stroboscopic motion analyzer system. The system was evaluated using high speed comb drive microactuators. Resonant frequency of $52.1 \mathrm{kHz}$ with a stroke length of $60 \mu \mathrm{m}$ was observed. Resonant motion of the combs at velocities of $20 \mathrm{~m} / \mathrm{s}$ was also successfully reconstructed from the imaging data. Small exposure times of $\sim 50 \mathrm{~ns}$ and the utilization of low camera frame rates enabled the capturing of sharp and bright images at optical spatial resolution of $0.5 \mu \mathrm{m}$. No other stroboscopic imaging system has been previously reported to image microstructures at such high speeds and frequencies, making this imaging system an inexpensive alternative to commercially available imaging systems.

Supplementary Materials: The video is available online at www.mdpi.com/2072-666X/8/12/351/s1.

Author Contributions: A.B. assisted in the fabrication of the microactuators and in writing and proofreading of the manuscript. M.U.K. contributed towards the development of the experimental set-up. His assistance in building the circuits was very instrumental in realizing the test set-up. C.H.M. helped throughout the testing and debugging of the circuits and also in preparing the manuscript.

Conflicts of Interest: The authors declare no conflict of interest. 


\section{References}

1. Lawrence, E. Optical Measurement Techniques for Dynamic Characterization of MEMS Devices. Available online: http:/ / www.polytec.com/fileadmin/user_uploads / Applications/Micro_Nano_Techno logy/Documents/OM_TP_MEMS_Whitepaper_2012_07_E.pdf (accessed on 6 September 2017).

2. Lawrence, E.M.; Speller, K.E.; Yu, D. MEMS characterization using laser doppler vibrometry. Proc. SPIE 2003, 4980, 51-62.

3. Davis, C.; Freeman, D.M. Statistics of subpixel registration algorithms based on spatiotemporal gradients or block matching. Opt. Eng. 1997, 37, 1290-1298. [CrossRef]

4. Davis, C.; Freeman, D.M. Using a light microscope to measure motions with nanometer accuracy. Opt. Eng. 1997, 37, 1299-1304. [CrossRef]

5. Smith, N.F.; Tanner, D.M.; Swanson, S.E.; Miller, S.L. Non-destructive resonant frequency measurement on MEMS actuators. In Proceedings of the 39th Annual IEEE International Reliability Physics Symposium, Orlando, FL, USA, 30 April-3 May 2001; pp. 99-105.

6. Rembe, C.; Muller, L.; Muller, R.S.; Howe, R.T. Full three-dimensional motion characterization of a gimballed electrostatic microactuator. In Proceedings of the 39th Annual IEEE International Reliability Physics Symposium, Orlando, FL, USA, 30 April-3 May 2001; pp. 91-98.

7. Hart, M.; Conant, R.A.; Lau, K.Y.; Muller, R.S. Time-resolved measurement of optical MEMS using stroboscopic interferometry. Proc. Transducers 1999, 99, 470-473.

8. Rembe, C.; Muller, R.S. Measurement system for full three-dimensional motion characterization of MEMS. J. Microelectromech. Syst. 2002, 11, 479-488. [CrossRef]

9. Hart, M.R.; Conant, R.A.; Lau, K.Y.; Muller, R.S. Stroboscopic interferometer system for dynamic MEMS characterization. J. Microelectromech. Syst. 2000, 9, 409-418. [CrossRef]

10. Rembe, C.; Tibken, B.; Hofer, E.P. Analysis of the dynamics in microactuators using high-speed cine photomicrography. J. Microelectromech. Syst. 2001, 10, 137-145. [CrossRef]

11. Guo, T.; Chang, H.; Chen, J.; Fu, X.; Hu, X. Micro-motion analyzer used for dynamic MEMS characterization. Opt. Lasers Eng. 2009, 47, 512-517. [CrossRef]

12. Petitgrand, S.; Bosseboeuf, A. Simultaneous mapping of out-of-plane and in-plane vibrations of MEMS with (sub) nanometer resolution. J. Micromech. Microeng. 2004, 14, S97. [CrossRef]

13. LM_BR_MSA-400.pdf. Available online: http://www.ects.pl/files/uploader/Producenci/Polytec/Wibro metry_do_pomiarow_mikrostruktur/MSA-500/LM_BR_MSA-400.pdf (accessed on 6 September 2017).

14. Lyncée Tec. Reflection Configured Digital Holographic Microscope $\left(\mathrm{DHM}^{\circledR}\right)$, a Non-Scanning and Non-Contact Method for Static and Dynamic 3D Topography as Well as Vibration Characterization. Available online: https:/ /www.lynceetec.com/wp-content/uploads/2014/02/LynceeTec_DHM_Rseries _Datasheet_2014.pdf (accessed on 6 September 2017).

15. 74HCT4059 Datasheet (PDF)—NXP Semiconductors. Available online: http://www.alldatasheet.com/datas heet-pdf/pdf/15613/PHILIPS/74HCT4059.html (accessed on 6 September 2017).

16. Tang, W.C.K. Electrostatic Comb Drive for Resonant Sensor and Actuator Applications. Ph.D. Thesis, University of California, Berkeley, CA, USA, 21 November 1990.

17. Zhou, G.; Dowd, P. Tilted folded-beam suspension for extending the stable travel range of comb-drive actuators. J. Micromech. Microeng. 2002, 13, 178. [CrossRef]

18. Dennis, J.O.; Ahmed, A.Y.; Khir, M.H.M.; Rabih, A.A.S. Modelling and simulation of the effect of air damping on the frequency and quality factor of a CMOS-MEMS resonator. Appl. Math. Inf. Sci. 2015, 9, 729-737.

19. Eltagoury, Y.M.; Soliman, M.; Sabry, Y.M.; Alotaibi, M.J.; Khalil, D. Electrostatic comb-drive actuator with high in-plane translational velocity. Micromachines 2016, 7, 188. [CrossRef]

(C) 2017 by the authors. Licensee MDPI, Basel, Switzerland. This article is an open access article distributed under the terms and conditions of the Creative Commons Attribution (CC BY) license (http://creativecommons.org/licenses/by/4.0/). 